\title{
Health Education and Media Literacy: A Culturally-Responsive Approach to Positive Youth Development
}

\section{Julian Owens $\mathbf{D}^{*}$ and Bradley Smith $\mathbf{H}$}

Johns Hopkins Bloomberg School of Public Health, Mental Health, Johns Hopkins University, 6023 Riddle Walk, Alexandria, Virginia, VA 22312, United States of America

${ }^{*}$ Corresponding author: Julian Owens D, Johns Hopkins Bloomberg School of Public Health, Mental Health, Johns Hopkins University, 6023 Riddle Walk, Alexandria, Virginia, VA 22312, United States of America, Tel: 7036243459; E-mail: julianowens@jhu.edu

Received date: Apr 13, 2016; Accepted date: Apr 27, 2016; Published date: Apr 29, 2016

Copyright: (C) 2016 Owens JD, et al. This is an open-access article distributed under the terms of the Creative Commons Attribution License, which permits unrestricted use, distribution, and reproduction in any medium, provided the original author and source are credited.

\begin{abstract}
The average American teenager spends nearly eleven hours a day engaged in social media and accessing digital music multimedia, which has negative effects on health and educational attainment. Curricula that contain youthfriendly media could improve learning, reduce risky and problem behavior and improve health outcomes, but may be controversial given some of the content. This study was designed to assess stakeholder's perceptions of a curriculum built around Popular Youth Music Media (PYMM) called Musics Energy: The Message in the Music ${ }^{\mathrm{TM}}$ (ME: MIM). This intervention consists of six modules implemented over 24 sessions. ME-MIM uses PYMM to teach students how to deconstruct music by collaborating, communicating, reading closely, thinking critically, conducting research and taking useful notes by listening. In-depth interviews and focus groups were conducted with 11 middleschool students and 6 teachers across multiple school districts. The constant comparative method was employed to explore emerging and recurring themes. Major themes were that ME: MIM was acceptable to most students and conditionally acceptable to most teachers. Stakeholders indicated that ME:MIM may be used as a component of a culturally informed, carefully monitored curriculum. Given the findings of this study, ME:MIM appears to be an acceptable and feasible approach to addressing health risk behavior, education disparities and educational engagement, especially among those who may be at higher risk for school disengagement and those who are exposed to more programmed media messages.
\end{abstract}

Keywords: Adolescents; Culturally-relevant pedagogy; Culturallyresponsive teaching; Culturally-familiar instruction; Culturallyengaging learning; Health literacy; Media literacy; Pop culture; 21st century competencies and youth culture

\section{Introduction}

Today's youth typically spend a significant amount of time engaged in entertainment media that may encourage risky or antisocial behavior $[1,2]$. Student-centered interventions that mitigate negative influences of popular youth music media [PYMM] may have significant personal and public health benefits [3,4]. Furthermore, harnessing the learning opportunities associated with the use of PYMM may re-channel student interests and have positive effects on education [5] health and critical thinking about media messages.

\section{Popular youth culture}

Popular youth culture (PYC) refers to what is "in" and resonating among most youth ages 11-25, irrespective of race or socioeconomic status. Hip-hop culture now dominates popular youth culture [6-8] and has been described by scholars as a "psycho-socio-culture" with its primary elements involving the reciprocal interaction of individual, societal and cultural forces [9]. Specific elements of popular youth culture include: how youth spend their time; what they value, their attitudes, behavior, choices about style; and how young people interact with mass media messages, their peers, and society at-large [9].

Some youth appear to be more at-risk for negative influences linked to PYMM than others. The effect of media varies by race and ethnicity, with the greatest effect on those who are heavily engaged with entertainment media. For example, based on the most recent study on media in the lives of 8-18 year olds conducted by the Kaiser Family Foundation, Black and Hispanic youth are more engaged with recreational media compared to their white counterparts [10]. Therefore, intervention and prevention programs targeting minority youth and designed to address school engagement, problem and risky behavior, and the achievement gap could be highly effective by thoughtfully integrating PYC and specifically PYMM into formal and informal learning spaces.

In addition to minority group membership, economic disadvantage and location may be additional risk factors for some youth. According to a recent Youth Risk Behavioral Surveillance Survey (YRBSS) [11] youth in some southern states are at greater risk for adverse health outcomes compared to other youth across the nation. Therefore, this research was conducted with key stakeholders in South Carolina.

There is a dearth of pedagogical instruction that addresses the prevailing themes and messages in PYMM. Therefore, it may be helpful to develop evidence-based interventions that leverage the broad appeal and pervasive influence of digital media in the lives of minority youth. This content may be a particularly effective way to engage youth in a dialogue about the precursors of behavior such as individual values, social norms, critical thinking and effective communication within the broader context of what researchers describe as popular youth culture $[6,8,9]$. Special attention to the themes and messages linked to health risks and problem behavior are critically important, as well as opportunities to address student engagement, school climate and culture. 
Harnessing the learning opportunities associated with the use of youth music media may have positive effects on education [5] and potentially very positive benefits in delivering culturally-relevant education. Evidence-based programs or interventions that mitigate negative influences of youth music media (i.e., lyrics, sound recordings and music videos) may have significant benefits $[3,4]$ including better student outcomes, more engaged parents and support from teachers. What is missing is an evidence based approach that emphasizes student competencies and works as a 'behavioral vaccine' to counteract negative influences of recreational media.

The present study is designed to test the acceptability and feasibility of a new youth-oriented media literacy program called Musics Energy: The Message in the Music ${ }^{\text {ta }}$ (ME:MIM). This intervention consists of six modules implemented over 24 sessions and uses PYMM to teach young people how to deconstruct music media by collaborating, communicating, reading closely for comprehension, thinking critically and taking useful notes based on listening. ME:MIM is being developed in the context of the deployment-focused [DF] model intervention development and testing. ME: MIM aims to: 1) improve communication linked to teaching, learning and student engagement and; 2) to address academic, behavioral, emotional, moral and social competencies linked to positive youth development.

The DF-Model [12] consists of a total of six steps. These are: 1) assessment and planning; 2) staff training; 3) supervised implementation; 4) monitoring implementation; 5) two-way feedback with program revisions; and 6) ongoing data collection. Step 1 in the DF model is to develop and assess acceptability and feasibility in "real life" settings. This step was the focus of this study and was intended to close the research to practice gap by creating interventions that "real life" providers will actually use [13]. Accordingly, two research questions were addressed in the present study: 1) Will teachers find music media literacy to be an acceptable and feasible approach to engage students and improve teaching and learning linked to stateadopted health education standards? 2) Will students who participated in the study find music media literacy an acceptable, feasible and satisfactory approach to enrichment programming that addresses health, education, communication and positive youth development?

\section{Music and social learning theories}

Learning through music occurs both formally and informally, as well as, intentionally and unintentionally. Perhaps of greater significance for the present study is that learning depends on the interaction of internal and external variables [14] that may include the setting, the facilitator, the content of material used for instructional purposes and the context of the instruction. During the middle school years and the transition to high school, many students may have not developed psychologically to the point of acquiring knowledge or "learning" that involves formal operations thinking. Researchers have found that if these students are supported in the development of critical analysis and critical thinking skills they may move through this period with fewer negative academic, behavioral, emotional or social outcomes. Promising methods for promoting this development are "hands-on" learning through the arts, and service learning.

The two major categories of learning theories that apply to music are: behavioral learning and social learning [14]. The behavioral theorists emphasize that external events cause changes in observable behaviors; that the outcome of learning is a change in behavior [15]. Social learning theorists acknowledge the external factors play a role in learning, however, they emphasize that learning also occurs through observing others and that learning is shaped by cognitive appraisal of events [16] thereby emphasizing the value of cognitive processing through dialogue and discourse analysis.

Substantive dialogue between and among youth and adults provides opportunities to analyze and deconstruct the themes and messages in music and to engage in critical analysis that may inform attitudes, beliefs and choices. For example, in a music video the characters may receive some form of reward or punishment as a consequence of behavior or a choice made. Without the opportunity to process or "deconstruct" this information, it may be difficult to reconcile it with one's own frame of reference or personal value system. This may be especially difficult for an adolescent whose brain is still developing up to age of 25. ME: MIM provides a forum for this discourse by having youngsters and adults who serve them collaboratively define and give examples of what each consider risky behavior, problem behavior, health risk behavior, and health promoting behavior. Additionally, young people and the adults who serve them also comment on the attitudes, beliefs, perceptions and scenarios that may prompted by deconstructing music media that influences choices and ultimately, to behaviors and habits.

To thoroughly address each risk area and promote generalization of pro-social skills, ME:MIM specifically addresses 10 risk areas. These risk areas are based on a content analysis study of 30+years of Grammy Award winning songs revealing that there are ten prevailing themes in popular youth music media. These themes are: 1) alcohol, tobacco and other drugs; 2) casual and suggestive sex; 3) criminal activity; 4) dark and deviant behavior; 5) profanity and foul language; 6) interpersonal conflict/bullying; 7) materialism; 8) narcissism; 9) social problems; and 10) violence, aggressive behavior and trauma. Of these ten themes, five are directly linked to risky behavior and may contribute to negative health-related outcomes. An ME:MIM example is lessons and activities that expose common advertising techniques used by media producers to attract consumers, especially young consumers' attention. These techniques include image appeal, which may be explicit or implicit images suggesting to consumers that "you will be more glamorous, sexy or macho if you smoke, drink or use drugs".

Black and Hispanic youth consume more media than their white counterparts. Interestingly, these youth in some southern states are more likely to be at-risk for depression and other serious mental illness [17] in comparison to their white counterparts. For these youth, some are more likely to: a) have felt sad and hopeless; b) have attempted suicide one or more times; or c) have made a plan about how they would attempt suicide [18]. Since racial and ethnic minority youth in the United States suffer an elevated risk for adverse mental health outcomes and these outcomes include depression and feelings of hopelessness, ME-MIM also addresses the importance of self-esteem and provides students healthy ways to deal with stress. For example, students engage in behavioral rehearsals that prompt them to critically examine antecedents that may lead to healthy and harmful outcomes; the emphasis being on choosing wisely based on known facts and lessons learned based on their own prior experience or the prior experience of others.

\section{Service delivery setting}

One approach to addressing the developmental needs of these students is to offer enrichment activities that extend learning opportunities beyond the traditional school day and during the summer. Extending the school day and providing summer enrichment learning opportunities has been found to offer protective factors that 
increases student's academic performance, especially when such programming is structured, cognitively stimulating ([19] and culturally relevant. Extending the school day and providing summer programming also appeals to parents/guardians and school officials because these programs may provide a safe place for students, help them improve their academic performance, and reduce behavioral problems such as juvenile delinquency. Many of the common challenges faced during the school day are avoided by extending the day via implementing approaches like ME: MIM.

After-school and summer programs provide a unique opportunity to offer structured programming that uses PYMM to assess how it may help students make informed decisions about avoiding problem and risky behavior and making pro-social, healthy choices. Given PYMM's immediate relevancy to the social and cultural context of adolescence, an intervention that leverages PYMM may yield insightful results that facilitate better-informed teaching and learning experiences for youth, especially those already on the verge of dropping out due to school disengagement. Moreover, such an approach highlights the need for culturally astute educators who are competent in PYMM and not intimidated by the content or context in which popular youth music and media exists and flourishes.

\section{The need for culturally competent educators}

Rap recording artists are pivotal to youth culture and these artists may play a bigger role than some would like in shape what future generation's value, as well as the collective consciousness, cultural norms and cultural pursuits [17]. A working knowledge of the culture that engulfs and contextualizes urban youth may help adults communicate more effectively with them. Additionally, it may be important to understand the information young people process (content) and helpful to use this information to ensure the (context) for linking what is taught to how it may apply inside and outside of educational spaces. This is especially so given the pivotal and sometimes negative role media may play in the lives of already highly vulnerable minority youth.

Harper [19] recommended that clinicians, educators and other youth-serving practitioners who wish to reach young people with their message or product work toward becoming culturally competent in popular youth culture. This recommendation is based upon the premise that cultural competence in popular youth culture is required to be effective, especially in educational spaces aimed at positive youth development. Moreover, researchers argue that those who use their knowledge and experience as the foundation for disseminating information and delivering services will likely succeed in reaching young people [6-8].

\section{Recreational media use and school expectations}

According to Rideout and colleagues [9], youth who spend more time with media report lower grades and lower levels of personal contentment. Heavy media users are more than twice as likely to report getting into trouble at school compared to light media users. Table 1 highlights that media has an even greater negative influence among black and Hispanic youth. Additionally, the CDC's 2011 Youth Risk Behavior Surveillance Survey [YRBSS] reports that black and Hispanic youth are more likely than white students across the U.S. to use computers for recreational purposes [17].

Table 1 below also illustrates two other important points: 1) black and Hispanic students are using every form of media more than their white counterparts; and 2) children of parents with less education use the media more than children of parents with some college or parents who have been educated beyond college.

\begin{tabular}{|l|l|l|l|l|l|l|}
\hline & \multicolumn{3}{|l|}{ Race/Ethnicity } & \multicolumn{3}{l|}{ Parents Education } \\
\cline { 2 - 7 } & White & $\begin{array}{l}\text { Blac } \\
\text { k }\end{array}$ & $\begin{array}{l}\text { Hispani } \\
\mathbf{c}\end{array}$ & $\begin{array}{l}\text { HS or } \\
\text { Less }\end{array}$ & $\begin{array}{l}\text { Some } \\
\text { Colleges }\end{array}$ & $\begin{array}{l}\text { College } \\
+\end{array}$ \\
\hline TV content & $3: 36$ & $5: 54$ & $5: 21$ & $4: 46$ & $4: 55$ & $4: 07$ \\
\hline Music/Audio & $1: 56$ & $3: 00$ & $3: 08$ & $2: 48$ & $3: 01$ & $2: 11$ \\
\hline Computer & $1: 17$ & $1: 24$ & $1: 49$ & $1: 28$ & $1: 40$ & $1: 24$ \\
\hline $\begin{array}{l}\text { Video } \\
\text { Games }\end{array}$ & $: 56$ & $1: 25$ & $1: 35$ & $1: 07$ & $1: 07$ & $1: 11$ \\
\hline Print & $: 39$ & $: 33$ & $: 34$ & $: 30$ & $: 30$ & $: 44$ \\
\hline Movies & $: 13$ & $: 43$ & $: 33$ & $: 32$ & $: 17$ & $: 23$ \\
\hline $\begin{array}{l}\text { Total } \\
\text { Media } \\
\text { Exposure }\end{array}$ & $8: 36$ & $12: 59$ & $13: 00$ & $11: 26$ & $11: 30$ & $10: 00$ \\
\hline
\end{tabular}

Table 1: In a typical average amount of time spent with each medium out of 24 hour day.

Given the rates of media use among some youth, education reform efforts may benefit from increased media literacy as an explicit goal of 21 st century teaching and learning. Media literacy is an active state of consciousness about media and its role in shaping perceptions. Specifically, media literacy is achieved when individuals understand the role of media in society and approach all media with an informed perspective about its influence in shaping perceptions. Individuals who are media literate acquire essential skills of inquiry and self-expression necessary to participate in a democracy [20]. Competency-based youth media literacy focuses on developing skills in youth that integrate feelings (emotional competence) with thinking (cognitive competence) and actions (behavioral competence).

The emphasis on competency in adolescents aligns with research that documents students' difficulty transitioning from middle school into high school. The ninth grade has been identified as the gateway for secondary school completion because many students get off track academically during their ninth-grade year [21]. Youth need to be engaged in school in ninth grade to develop the competences to progress through secondary education and beyond.

\section{Research Methods}

The present study was action research as defined by Reason and Bradbury [22]. This study also followed Step I of Weisz [12]. Deployment-Focused Model of Intervention Development and Testing [DF-Model]. The action research cycle, as proposed by Coughlan and Coghlan [23] consists of three main steps: a) to understand the context and purpose of the study; b) six 'mini-steps' to gather feedback, analyze data, plan, implement and conduct evaluation; and c) a meta-step to monitor. The DF-Model [11 consists of six steps: 1)assessment and planning; 2) staff training; 3) supervised implementation; 4) monitoring implementation; 5) two-way feedback with program revisions; and 6) ongoing data collection. A major part of step one of the DF-model is on ascertaining "real world" provider acceptability and perceived feasibility of the intervention. Completing Step 1 in both 
the Action Research Cycle and the adapted DF Model was the focus of the present study. Data gathered is used to further develop and refine the intervention starting with assessing its feasibility and acceptability among practitioners in natural settings.

PYMM may be an ecologically-valid means to engage students in social learning that reinforces key competencies linked to literacy, and maybe even health. Aligning the classroom with real world environments may result in a more engaging, culturally-responsive, inclusive learning space for all. An applied approach to learning may be particularly appealing to youth from diverse socio-cultural and racial backgrounds as this may give them more motivation to learn in a context that may have immediate relevancy to their lives. This could also align with state-adopted standards that may appeal to some administrators and parents.

The intervention examined in this study is MusicsEnergy: The Message in the Music (ME-MIM) and it addresses the following three state-adopted standards in South Carolina: Health Education Standard 2: "The student will analyze the influence of family, peers, culture, media, technology, and other factors on health behaviors" [24]. Media Arts Standard 3: "the student will access, analyze, interpret, and create media texts"; and Media Arts Standard 4: "the student will make connections between music, other art disciplines, other content areas, and the world" [24].

ME: MIM is an integrated, standards-driven approach to teaching and learning that engages students in individual and group activities reinforcing competency-focused positive youth development aimed at engaging youth, while increasing their health and media literacy. ME:MIM uses the three forms of popular music multimedia (i.e., song lyrics, sound recordings and music videos) to facilitate critical thinking and other 21 st century skills by deconstructing and discussing the themes and messages in music and the influence of these themes and messages on the attitudes, beliefs and choices linked to perceptions and ultimately to behavior. This integrated approach targets key competencies required for students to thrive in school and to be successful beyond school. Specific competencies addressed include: a) collaboration; b) close reading for comprehension; c) communication (oral, written and listening); creative expression; d) critical analysis (i.e., critical viewing and critical listening); e) information-seeking via research; and f) notetaking. This study is an evaluation of ME:MIM; the assessment was done via action research with students, in-depth interviews with teachers and surveys assessing satisfaction with the intervention.

\section{Data collection procedures}

From June 2014 to October 2014 in Columbia, SC, an action research study was conducted with a sample of parents, guardians, teachers, school administrators, program staffers and students $(n=24)$. Results reported in this manuscript were obtained from students and teachers only. The student sample was drawn from participants in the Challenging Horizons Program [CHP] for the 2014 academic school year, which included summer 2014. This population consisted of all students; 65 students of African-descent; 10 white; 6 bi-racial and 1 Latina. A total of 11 black middle school graduates were screened to be at high risk for adverse academic, social, and emotional outcomes. These students received a five-week version of ME: MIM. Additionally, 6 in-depth individual interviews were conducted with teachers and two $(n=2)$ focus groups were conducted with students. Students also completed a satisfaction survey.
Data collection procedures began by verifying that consent forms were signed by students' parent or guardian granting permission for participation in research consistent with the goals and objectives of the CHP. Permission to audio record students was granted via a signed release provided by the CHP as part of its approved Institutional Review Board (IRB) application at the University of South Carolina. Following each focus group, the transcript from the audio recordings was closely analyzed to identify emergent themes and patterns [25] based on responses to the semi-structured interview guide.

\section{Focus groups}

Two 60-minute student focus group interviews were conducted over the course of five weeks. The first student focus group was conducted after completing 12 days of programming (T1). The second student focus group was conducted at the conclusion of five weeks of programming (T2). The Participant/Researcher (P/R) documented students' responses to questions concerning whether they thought ME:MIM would: a) help them be more engaged in learning; and if they thought ME: MIM would help them: b) develop (or reinforce) academic, behavioral, emotional, moral and social skills required to be successful in school. Additionally, the $\mathrm{P} / \mathrm{R}$ documented students' perceptions about teachers and administrator's openness to using popular youth music media to facilitate a better teaching and learning in the classroom, specifically as it relates to critical thinking, collaborating and communicating with others. By following the interview guide, students and teachers addressed the research questions which helped illuminate other related topics, issues, priorities and concerns.

All focus group interviews were conducted in person with the $P / R$ and a co-facilitator. All individual and group interviews conducted by the $\mathrm{P} / \mathrm{R}$ were audio recorded. The $\mathrm{P} / \mathrm{R}$ was intentional about generating discussion regarding media and its role and potential influence in shaping attitudes, beliefs, perceptions, preferences, behavior and underlying values. This enabled the $\mathrm{P} / \mathrm{R}$ to assess if attributes of ME:MIM were acceptable and feasible and if so, under what conditions. Additionally, the P/R sought students' feedback about the use of PYC and specifically PYMM for instruction and enrichment purposes linked to engagement and school success.

\section{Interviews}

Teachers who teach select subjects implicated in ME:MIM were recruited by making phone calls to local schools in the greater Columbia area. A total of six $(n=6)$ teachers agreed to be interviewed. There were three health education teachers, one English Language Arts teacher; one Social Studies teacher and one Visual and Performing Arts/Media Arts teacher. The one-on-one interview format enabled the $\mathrm{P} / \mathrm{R}$ to capture authentic concerns about PYC and PYMM, as well as stakeholders' attitudes, beliefs and dispositions toward PYC and PYMM being used in schools. Interviews lasted from 50-60 minutes each. For the focus groups discussions and individual interviews, broad questions about PYC and PYMM were followed by specific questions using the interview guide. Probing questions were asked for additional clarification. Lively discussions ensued.

\section{Acceptability and satisfaction surveys}

Descriptive data from students and teachers was collected and analyzed. The following six potential outcomes were identified based on the research questions: 1) acceptability- assessed to be agreeable or 
satisfactory; 2) conditionally acceptability-assessed to be agreeable or satisfactory with conditions; 3) feasibility-capable of being achieved or possible; 4) ambivalence - the coexistence and simultaneous existence of opposing attributes or feelings; 5) acceptability and feasibilityassessed to be both satisfactory and possible; and 6) not acceptability/not feasibility-assessed to be neither satisfactory NOR possible.

In addition to audio recordings of the student focus group discussions, students also completed an acceptability/satisfaction survey to assess specific program components of ME:MIM, as well as to provide feedback about the use of PYMM and PYC for enrichment or instructional purposes.

\section{Data Analysis and Reporting}

\section{Content analysis, open coding, thematic analysis and saturation}

All focus group interviews and in-depth interviews were audiorecorded and professionally transcribed. This facilitated the excavation of emergent themes and relevant constructs [26]. A preliminary codebook was developed through an open coding process. Open coding of the transcripts led to the conceptual organization of the data based on potential themes. Open coding is the process of separating and examining data into patterns or themes, categorizing and linking data conceptually.

The codebook was finalized via a process that required re-coding all transcripts several times which is referred to in the literature as the axial coding process $[27,28]$. Axial coding enabled the identification of any thematic relationships that existed between codes [29]. All data were analyzed via thematic analysis, which breaks the text into relatively small units of content and submits these narratives to descriptive treatment [30]. The P/R then identified common threads across multiple interviews with students and teachers. Content saturation was reached by completing a meticulous process of establishing categories and subcategories. This was followed by connecting categorical properties and dimensions and then, using all interview data and identifying how the categorical properties and dimensions are related to develop themes [29]. Layering the data over time, gathering information from a variety of participants, and looking to more than one form of documentation helped validate data collected [31,32].

\section{Verification of validity and reliability}

Codebook categories, sub-categories, dimensions and definitions were updated after each transcript was analyzed and before proceeding to the next. Authors of this manuscript acknowledge the potential for bias toward the data, but incorporated the P/R's identity as a stakeholder during analysis. Doing so authenticates the P/R's own voice during interpretation of the participant's perspectives [25,26]. Every effort was made to faithfully record and interpret data from the participants' perspectives. In so doing, the P/R refrained from judging participant's data against his own value system or any other.

\section{Results}

Results obtained from students and teachers are reported according to the following four outcomes: 1) Acceptability; 2) acceptability/ feasibility; 3) conditional acceptability/ conditionally feasibility; and 4) not acceptable/ not feasible. These results are further interpreted through select theories that illuminate their potential to inform education and social policy and practice. For example, the Social Cognitive Theory (SCT) emphasizes that learning occurs through observing others. In this view people learn by watching others. This theory suggests that individuals learn from direct experience and from behavior modeled by others, which can occur via the media [22].

\section{Outcome: Acceptability/Feasibility}

School teachers: Representative responses from school teachers to the following five questions indicate acceptability/feasibility of the proposed approach.

Q1: Do you believe it is important to use media like what we have just discussed to engage students in classroom instruction? Please elaborate.

"One of my favorite quotes of all time is 'if they aren't learning the way that we teach we need to teach the way they learn, and that goes for what they're learning. We have to adapt how we're presenting our material based on what the world is presenting to them."

The Theory of Multiple Intelligences illuminates this statement because it acknowledges that intelligence is not a single variable to be measured only one way. Rather, intelligence is an innate potential in everyone activated by environmental cultural values, opportunities, experiences, and personal decisions [33]. Since music is one of the seven types of intelligences originally specified in this theory, it, along with interpersonal and intrapersonal intelligences forms a solid foundation upon which to implement more pro-social learning linked to music in both formal and informal learning contexts.

Q2: In your opinion, do you believe using popular youth music media for instruction would engage students in addressing select standards in C/IT, ELA, Health Ed, Social Studies and Visual and Performing Arts/Media Arts?

"I am very interested in Musics Energy because a very key part, in fact the major part of this grant is arts' integration into the classrooms and how does that affect diversity and student achievement."

"The things that they love, that they like and that they are drawn to for some reason, you know, why is it that they are pulled into those things and why aren't we addressing those? All of the things that you discussed are things that we want to address. We want to address their emotional and mental health."

The Sociocultural Theory of Learning illuminates this statement because it posits that social interactions, in this case social interactions between students and teachers, are critical for intellectual development; that learning, both in formal and informal contexts, is embedded within social events. This theory asserts that learning occurs as children interact with people, objects, and events in the environment [34].

Q3: Do you believe parents, school administrators and other school personnel would approve of using popular youth music media for classroom instruction if the instructional materials are approved prior to implementing in the classroom?

"The way this is being presented is religion is not a part of this. It's simply values; it's simply good, solid values that, if people live by this they have substance in their life and they have some purpose in their life as opposed to what is being portrayed that is certainly not." 
Observational learning is the theoretical construct of the SCT that asserts people can witness and observe the behavior of others, and then reproduce those actions. This is often exhibited through behavioral "modeling". If individuals observe a behavior, they have the capacity to replicate that behavior just by merely observing it [35]. Observed behavior may be even more appealing when coupled with standard advertising techniques and media attributes commonly used in popular media that appeals to young people.

Q4: In your opinion, do you believe using youth-oriented popular music media for instruction would engage your students in addressing select standards listed more than the curriculum that is currently being used?

"I definitely think what is being proposed here can be used to address standards. It is something that, because you are using the message and you're using what the kids are familiar with, that's the hook to get them to become interested."

Lev Vygotsky's Sociocultural Theory of Learning apples to this statement given it posits that social interactions are critical for intellectual development, especially when these interactions reflect what is culturally relevant, culturally responsive and culturally familiar to the target audience. Again, learning is embedded within social events and occurs as children interact with people, objects, and events in the environment [34]. Additionally, Jean Piaget's Theory of Cognitive Development is relevant to this statement given posits that children and adults think differently [36], and this may be especially so when it comes to age differences, perceptions about popular youth culture and perceptions linked to popular youth music media.

Q5: What is needed to enable you to feel better prepared to integrate the concept of media literacy into your lesson plans?

"I just think a bank of songs and lyrics, would be helpful cause so many of the lyrics are inappropriate but that would be a very useful tool for teachers because part of the time we don't have time to, you know; build a lesson plan around something or to make a whole new lesson."

The theoretical implications of the spiral curriculum [37] may be relevant in this statement given that it suggests if teachers use music for pedagogical purposes it should be with larger objectives in mind for students to learn. The spiral curriculum places great importance on teachers carefully planning how they will present material to get the greatest yield from the time it takes to deliver instruction [37]. Instruction may include music, placing even greater importance on students' cognitive development and the sociocultural context in which teaching and learning occurs. Attention to detail, as well as the larger objectives lays a solid foundation for scaffolding and the transference of information and skills from one situation to another. Transference from concept to application is essential and integral to Bloom's Taxonomy of Learning which is the classification system used to define and distinguish different levels of human cognition, for example thinking, learning, and understanding. Since, most educators indicate instructional time is limited in the formal educational setting, maximizing the amount of quality time and interaction that a child has with his or her teacher is highly desirable. Having a tool that enables educators to quickly retrieve relevant media for instructional purposes would increase the amount of quality instructional time.

Students: One hundred percent (100\%) of the students surveyed indicated that they would recommend ME:MIM to their best friend. Ninety percent (90\%) indicated that they would recommend ME:MIM to their peers; eighty $(80 \%)$ indicated that they would recommend ME:MIM to their teachers or administrator, and seventy percent (70\%) indicated that they would recommend ME: MIM to their parent/ guardian. Of all students surveyed, one-hundred percent (100\%) indicated that they found ME:MIM to be highly engaging and ninety percent (90\%) indicated that they believe ME:MIM should be offered during the school day. Sixty percent $(60 \%)$ indicated a preference for ME:MIM being offered both during the day and after school. Additionally, eighty percent $(80 \%)$ of students surveyed indicated that they believe ME:MIM is a culturally relevant and culturally familiar approach to learning in a social setting.

Detailed responses from students about the link between PYMM and their attitudes, behavior, choices, feelings and values are found in Tables 2, 3 and 4 .

\begin{tabular}{|l|l|}
\hline Behavior in school or out of school & $100 \%$ \\
\hline Attitudes and beliefs about school & $100 \%$ \\
\hline Feelings about how to treat others & $90 \%$ \\
\hline Values related to health risks & $80 \%$ \\
\hline Values related to problem behaviors & $80 \%$ \\
\hline Choices linked to health risks & $80 \%$ \\
\hline Choices linked to problem behavior & $80 \%$ \\
\hline Feelings in general & $80 \%$ \\
\hline
\end{tabular}

Table 2: Percent of students who indicated that they understand the link between PYMM.

\begin{tabular}{|l|l|}
\hline Best friend & $100 \%$ \\
\hline Peers & $90 \%$ \\
\hline Parent/Guardian & $70 \%$ \\
\hline Teachers or Administrators & $80 \%$ \\
\hline
\end{tabular}

Table 3: Percent of students who would recommend ME-MIM.

\begin{tabular}{|l|l|}
\hline Is engaging & $100 \%$ \\
\hline Should be offered during the day & $90 \%$ \\
\hline Is culturally relevant and culturally familiar & $80 \%$ \\
\hline Should be offered only after-school & $40 \%$ \\
\hline
\end{tabular}

Table 4: Percent students who believe ME-MIM.

Outcome: Conditional Acceptability/Conditional Feasibility

School teachers: Representative responses from school teachers to the following five questions indicate conditional acceptability/ conditional feasibility of the proposed approach.

Q1: Do you believe it would be helpful to use culturally relevant, cultural familiar media to engage students and motivate learning in the classroom? 
"Yes, in lower income school districts and settings, to start off with then move toward middle income schools and settings to maybe move into upper income....

There's the thought, if it can work with [inner city schools that serve inner city youth], it can work with anybody... So it's kind of like it works in the hard core areas it can work in the areas that are changing like ours."

Critical Race Theory (CRT) is strongly implicated in this statement. $\mathrm{CRT}$ is a theoretical framework in the social sciences that begins with the notion that racism is normal in American Society. It focuses on the application of critical theory, a critical examination of a society and its culture, and the intersection of race, law and power. CRT recognizes that race-based biases and discrimination is deeply engrained in the fabric and the education, political and social systems that make up American society.

Q2: In your opinion, what is the main challenge in using popular youth music media as instructional material in the classroom?

"We still have administrators that are predominantly traditional Eurocentric, 'this is how we do it', you know, 'we're still in 1975', you know. Your really engaging teachers might feel like they have to pull back because of that so there's a lot of dynamics in any public school or any school situation for sure."

Expectations, a construct of the SCT, refer to the anticipated consequences of a person's behavior. In other words, a person who anticipates the consequences of his or her actions before engaging in a behavior is doing so based on some sort of expectations. The anticipated consequences of one's behavior may influence successful completion of the behavior. Expectations are derived largely from previous experience. While expectancies also derive from previous experience, expectancies focus on the value that is placed on the outcome and are subjective to the individual [15]. In this case, a teacher's expectations and his perception of the administrator's expectations may directly influence his willingness to invest personally in such an approach.

Q3: Do you believe other stakeholders (e.g., parents, students, school administrators, counselors, community members and other staff) would approve of using popular youth music media for classroom instruction if all materials are approved prior to implementing in the classroom?

"To me, South Carolina needs to have this; it's going to be more accepted, especially when there's some studies, you know... Or until you have somebody that's really willing to ride out on the edge of the frontier with this".

Again, behavioral capability is the theoretical construct of the SCT that refers to a person's actual ability to perform a behavior through applying essential knowledge or skills. In this case, one may infer that stakeholders receive cues about how to apply essential knowledge or skills based on the consequences of their behavior or the behavior of others within the school environment. Their behavior is directly correlated with what they have learned and their behavioral capability is enhanced or constrained based upon how they interpret what they learn and any cues from the environment that influence their perception [15].

Q4: What is needed to enable you to feel better prepared to integrate media literacy goals and objectives into your current lesson plans and would help you develop new ones?
"You have to be instructor who sees their role more as a Socratic process where you're more facilitated in allowing - by asking questions, by allowing the kids to come to their own conclusions rather than, I want to go in and change them".

Q5: In your opinion, why are more teachers not using popular youth culture and entertainment media to engage students in in classroom instruction?

"The teacher [who uses PYMM for instruction purposes] has to know that the person above them is going to protect them and is going to understand what is going on because without the administrator then the teacher is putting themselves at risk for losing their job."

Behavioral capability is the theoretical construct of the SCT that best fits the three previous responses from teachers. This construct refers to a person's actual ability to perform a behavior through essential knowledge and skills. In order to successfully perform a behavior, a person must know what to do and how to do it. People learn from the consequences of their behavior, which also affects the environment in which they live, or in this case the environment in which they work.

Students: Representative responses from students to the following three questions indicate conditional acceptability of the proposed approach.

Q1: Why do think your peers may not want to have discussions about media's influence on their thoughts and behavior with teachers?

"I'm not interested in having a discussion if it's [with] one of those teachers that just don't care....if she act like she don't care it changes everything."

"It depends on how she talks about it...like how you approach it...it depends on if the teacher is cool or not."

Critical Race Theory is a theoretical framework in the social sciences that begins with the notion that racism is normal in American Society. It focuses on the application of critical theory, a critical examination of a society and its culture, and the intersection of race, law and power.

Q2: Would you be more engaged if your teachers used the media you like to develop lesson plans?

"It depends [on] if they like that type of music....I don't want to [talk about the music I like with my teacher or staff] if it's going to be negative...so that's like [attempting to] change the audience's mind about the music...It depends on how you talk about it....like how you approach it."

Q3: In your opinion, do you believe teachers would be comfortable using popular youth music media, for example, hip-hop songs and videos in the classroom? If not, why do you think they may not be comfortable?

"Sometimes they can be racial and they call it Black people music and some people don't like some students and they ignore them... Because some of the artists we like didn't go to school and they think we might end up dropping out of school."

As previously stated expectations refer to the anticipated consequences of a person's behavior. For example, students may anticipate the consequences of their actions before engaging in the behavior, In this case, students my anticipate negative consequences of permitting teachers to discuss the music and media they enjoy which 
could be an obstacle to using this approach. Anticipated consequences, or expectations, can influence successful completion of the behavior, especially since expectations are derived largely from previous experience. Expectancy is closely related to expectations in that they also derive from previous experience. Expectancy, however, focuses on the value that is placed on the outcome and is subjective to the individual.

Additionally, CRT is strongly implicated in this statement. One of the earliest themes within CRT was epistemology which begs the question: "why are people of color more likely than white people to see American society as racists?" A similar question is "why are people of color more likely than whites to be pessimistic about addressing racism or the possibility of eradicating racism in society?" These statements reflect an epistemological perspective expressed by one young black male respondent. CRT recognizes that race-based biases and discrimination is deeply engrained in the fabric and system of the American society.

\section{Outcome: No Acceptability/No Feasibility}

School teacher: There was one school teacher who indicated a lack of acceptability/feasibility of the proposed approach. Representative responses from this respondent to the following three questions are noted.

Q1: What do you think about using culturally relevant, culturally familiar media like websites of song lyrics, sound recordings and music videos in the classroom?

"Many teachers think some of the PYMM that students like is simply inappropriate. Aside from that, it's not to them what they think they should be teaching".

Q2: In your opinion, what is the main challenge in using popular digital music multimedia as instructional material in the classroom?

"If you want to teach them research skills in middle school and they don't want to do research; oh well you get to research your favorite artist - oh cool. Yea, but we're way past that we're in a totally different realm. It's not, 'oh honey, well you don't like this topic, it's okay'.

No, not to get them into college. I mean if you look at the lyrics of some of these songs, it's grammatically incorrect; some of them if you go and print out the lyrics they use texting abbreviations. No."

Q3: What do you think about using culturally-relevant, culturallyfamiliar popular music media as an approach to engage students in classroom instruction?

"A Lil John lyric showed up in our Valedictorian speech graduation speech last year. Yes, it's there but it's not how we teach them. Yes it is in the children's lives, but we don't teach through that."

Reciprocal determinism is implicated in each of these four responses; it explains the interactions between a teacher, her students and her role in setting the tone for the school environment, specifically in her classroom. In this case, students' behavior is controlled or determined by: a) the way the teacher cognitively processes; and b) by the environment within her classroom, through her interpretation of external social cues.

\section{Adolescence, social learning theories and music}

There are several important implications from this research about using popular youth music as content and context for social learning among adolescents, and specifically how social learning and human development theories informs these findings. For example, the theory of cognitive development emphasizes that adults need to better understand how children think in order to match teaching with children's abilities and interests at the appropriate stage of their development. This has implications for teachers and parents with regard to their teaching methods and parenting practices and tactics, respectively. Music plays a central role in the lives of adolescents, specifically as it relates to identity formation.

Importantly, the significance of the sociocultural theory of learning for teachers is that curriculum and instruction would appeal more to young people if teachers were more intentional about collaborating with young people about how to apply knowledge gained from schooling to real world scenarios, specifically the content and context of the instructional material used in formal and informal settings. In so doing, teachers would ensure students are engaged in the learning experience and this would ensure that what is being taught is relevant to the students' social and cultural real world experiences.

\section{Discussion}

This study was designed to access the acceptability and feasibility of bringing the potentially sensitive topic of PYMM into the classroom using the ME-MIM curriculum. Overall, teachers and students, found the approach to acceptable and feasible. A detailed interpretation of each stakeholder's perspective is presented below, including factors that may impact implementation success.

\section{Teacher satisfaction and insights}

The majority of teachers found ME-MIM to be an acceptable approach to teaching and learning for enrichment or instructional purposes. Teachers' comments indicate that they found ME-MIM to be culturally relevant, culturally engaging, culturally responsive and student-centered. Teachers also noted that ME-MIM would be culturally familiar to most students and a novel pedagogical approach to teaching; that ME-MIM could reach youth already at higher risk for dropping out of school or those who may otherwise not be engaged in the traditional school curriculum.

Teachers across multiple interviews consistently reiterated three points. First, ME-MIM is a pedagogical adaptation of PYC and PYMM; all of the teachers acknowledged the role of culture and media in the lives of today's youth, especially those in urban centers across the United States. Teachers also reiterated that ME-MIM is an approach that both students and teachers would appreciate, noting that MEMIM may be particularly appealing in school districts that are working toward integrating arts in the classroom and interested in assessing its effect on diversity and student achievement. Secondly, teachers consistently pointed out that ME-MIM's emphasis on health, behavior and media literacy aligns directly with the health education standards and that it may be one way to address students' emotional and mental wellbeing, as well. Thirdly, teachers noted that using PYC and PYMM as content and context for classroom instruction and enrichment programming subtly introduces ethical considerations and moral reasoning without being direct; that doing so through music facilitates a judgment-free dialogue with young people who are developing their own values.

The data from teachers is categorized as follows: 1) curriculum and instruction; 2) diversity and student achievement; and 3) student support services. In addition to teachers' comments that ME-MIM is student-centered and engaging, they also noted that an additional 
attraction of this approach to teaching and learning is its link to stateadopted national standards in multiple subject areas, as well as, that is implemented in a modular format that is curriculum-driven. Teachers also noted that there are federal, state and private grant-sponsored initiatives that seek to address diversity and student achievement through the arts and that ME-MIM aligns well with these funding opportunities, as well as, a number of recent educational priorities. Finally, teachers alluded to the topic of student's gaining moral competence through clarifying their values. Moral competence is one of the five competencies integrally tied to positive youth development. It can be controversial in and of itself, but highlights the need for support services and enrichment opportunities for students to address moral reasoning, in relationship to emotional and mental health and wellbeing.

Although most of the data from teachers indicate ME-MIM is acceptable and feasible, these data also provide insight about the conditions and factors impacting acceptability and implementation. Three themes emerged across teacher data: 1) socio-cultural/socioeconomic considerations; 2) administrator considerations; and 3) facilitator/teacher considerations. First, there was the sentiment among teachers that the ME-MIM would be more acceptable and feasible in a lower income school district initially and could be potentially be implemented in an upper income school or district afterwards. The exact quote from one teacher was: "if it can work with the inner city schools that serve inner city youth, it can work anywhere with anybody....and in the areas that are changing like ours." The sociocultural/socio-economic implications of teacher's comments are strong. This teacher's biases, conceptions and preconceptions would likely impose significant implementation limitations. Such sentiment is particularly illuminating in schools where administrators and personnel begin with racialized perceptions about urban youth. This sentiment is best summarized in this direct quote: "we still have administrators that are predominantly traditionally Eurocentric....your really engaging teachers might feel like they have to pull back because of that...there're a lot of dynamics in a public school for sure." Conversely, another teacher noted that it is important for the teacher/facilitator to view his or her role as Socratic - meaning; the emphasis in the activities or lesson plans is weighted toward skillfully raising questions and challenging participants to considering alternative points of view. This highlights the need for the facilitator to identify his or her own teaching philosophy or teaching style because it could impact the delivery of ME-MIM with fidelity.

There was one teacher who did not find ME-MIM to be acceptable or feasible. Data from this teacher maps onto the general theme that ME-MIM lacks the pedagogical rigor for a college-bound student. This assertion directly negates previous comments highlighting the link between ME:MIM and its positive correlations to curriculum and instruction. Additionally, this perspective is contrasted by data collected from students who emphasized the importance of the teacher withholding their own aspersions about PYMM and PYC. Furthermore, students are clear that their willingness to engage in an honest dialogue about PYC and PYMM in school "depends on how she [the teacher] talks about it...like how [she] approaches the topic." This emphasizes the importance of youth having a relationship with a caring adult or teacher that they perceive to be judgment free.

\section{Student considerations}

Student Satisfaction: Students assessed ME-MIM to be conditionally acceptable while also rating it highly satisfactory. In general, students expressed that they like this approach to teaching and learning, and they indicated that they believe it could help them be successful in school. For example, student satisfaction scores indicated that eight out of ten students understand the link between PYMM and its influence on their attitudes, beliefs and behavior in and out of school. Most students also indicated that they see how PYMM may influence the way they treat others. However, only six out of ten students indicated that they believe ME-MIM prompts them to think more critically about music and its influence in their live. Thus, only a slight majority of students indicated that they see the relationship between PYMM and their values as it relates to healthy decision making, risky behavior and other problem behavior. These findings are consistent with the conclusion that ME-MIM is acceptable to most students under certain conditions, but additional efforts may be needed to effect behavior change.

There were two primary themes extracted from the qualitative data collected from students. The first was that the relationship with the facilitator (e.g., teacher or staff) is pivotal. This has direct implications on curriculum and instruction during the school day and student support services that extend beyond the traditional school day. The main takeaway, regardless of the time of day, is that relatability of the adult facilitator may make the difference regarding the success (or lack thereof) of an approach that leverages PYC or PYMM. The second theme was that adult biases, conceptions and preconceptions may be a confounding factor in implementing ME-MIM with fidelity, unless fidelity measures are explicit and honored. One student noted that "sometimes they [teachers] can be racial and they call it black people music and some people don't like some students and they ignore them...because some of the artists we like didn't go to school and the think that we may end up dropping out of school."

Song lyrics, sound recordings and music videos are an integral component of ME-MIM. These musical attributes are consistent with what teachers and students believe to be culturally-relevant content that may be used to address standards linked to five content areas offered in the middle and high school curriculum. These content areas are Health Education, as well as Computer and Information Technology, English Language Arts, Visual/Performing Arts/Media Arts, and Social Studies. Students emphasized the importance of the adult facilitator refraining from even the perception of being judgmental as this would restrict the open dialogue and two-way communication that is integral to the design and delivery of ME:MIM.

\section{Teacher success factors}

A key factor in the successful uptake of ME:MIM is that teachers must have clearly laid out goals and objectives linked to specific lesson plans and activities and coupled with useful tools to ensure that such an approach to teaching and learning is successful. Additionally, teachers indicated that they need some idea about what to expect when allowing access to popular youth music media, especially when used for enrichment or instructional purposes. For example, teachers indicated that there would need to be more emphasis on cultural diversity and social justice pedagogy in pre-service training, specifically in their teacher education programs, if ME-MIM or a similar approach were to be accepted. Current teachers would need opportunities to gain the requisite knowledge, skills and abilities via professional development and/or training in order to become familiar and comfortable using PYMM as intended. In instances where teacher education programs do not offer the required learning opportunities and supports, school administrators, department heads and others 
responsible for training may need to supplement formal education experiences with targeted in-service training.

\section{Student success factors}

Student success is facilitated through interpersonal relationships with a caring adult. These relationships are especially important during the middle to high school transition and throughout high school. It is also critically important to create a sense of belonging or community to attract and sustain students' interest and motivation to learn. Facilitating academic and social success in schools may depend upon two factors: 1) access to a culturally-enriching, culturally-responsive curriculum; and 2) access to professional teachers and trained staff who are properly prepared to meet the needs of all children, regardless of their differences, and sometimes because of these differences.

Student and teacher stakeholders indicated that PYMM may facilitate enrichment programming and instruction because the content is immediately familiar, relevant, and engaging to young people. The person facilitating the discussion, however, must be viewed as trustworthy and Socratic in his or her approach to teaching and learning. PYC and PYMM may be a critical component of a culturally informed, carefully monitored standards-based, curriculum-driven intervention, particularly among those who are exposed frequently to popular youth media and programming targeting these young consumers.

The present study was the first to document student and teacher's assessment of ME:MIM. A few key themes that emerged among students and teachers are: 1) the need to address equity and social justice issues in a Socratic manner; 2) the need for cultural sensitivity and a genuine respect for multicultural diversity; 3) a willingness to acknowledge, despite how offensive some cultural norms that prevail in popular youth culture and music may be; and 4) a willingness to face one's own biases, conceptions and preconceptions in relation to the prevailing themes in PYC and PYMM. Based on qualitative and quantitative data gathered, successful uptake of ME:MIM requires culturally sensitive providers who have demonstrated a fundamental understanding and appreciation for cultural diversity, social justice and youth culture.

In summary, the use of PYC and PYMM for enrichment programming or instructional purposes appears to be helpful in the following three ways: 1) to engage students in social learning; 2) to address national and state-adopted standards to improve health and media literacy; and 3) to reinforce competencies linked to positive youth development and school success by addressing education and disparities. Data suggests that students and teachers found that popular youth music media (PYMM) provides the content and popular youth culture (PYC) provides the cultural context for new approaches to teaching and learning via enrichment programming and possibly curriculum and instruction that directly embraces the deconstruction of messages in PYMM.

\section{Limitations}

This qualitative study explored the acceptability and feasibility of using popular youth culture and specifically popular youth music media as an approach to teaching and learning. As such, there was no need for a large number of participants or a large sample that could be considered more representative of the general population. While there is no attempt to generalize these results, information gathered from this sample may be representative of other similarly-situated stakeholders. The small sample size is therefore not considered a limitation in the traditional sense, but may be considered an attribute to the study design at this particular phase of research.

Selection bias may also be considered a limitation when viewed in the traditional sense, as well as, limited exposure to the curriculum content by teachers. Another potential limitation is that student participants were screened based on inclusion criteria set by the Challenging Horizon Program. Consequently, these results may only apply to a similar population. This is not viewed as a significant limitation given the study authors' interest in working with youth who may be marginalized based on race and social class. Another limitation is that this study provides little details about which features ME:MIM's six modules appeal to the stakeholders. Student stakeholders, however, did provide feedback, albeit limited, about each module. And finally, teachers did not directly participate in ME:MIM activities/lessons, hence their comments were limited to the description of the pedagogical link between ME:MIM and three South Carolina-state adopted standards in Health Education and Media Arts, which are referenced above.

\section{Translation education policy and practice}

The scope of the present study focused only on the acceptability and feasibility of ME: MIM as an approach to teaching and learning. Quantitative and qualitative data generated from this study can be used to refine methodological procedures and address limitations in a follow-up study. For example, more attention should be given to developing a song bank for instructional purposes as this would facilitate standardizing instruction, while also giving student and teachers the desired flexibility for song selection and deconstruction. Data also suggests these songs should be tagged with relevant themes to expedite instruction and enable more standardized, yet flexible instruction and fidelity. Collection of quantitative data on student outcomes from baseline to post intervention was beyond the scope of the present study; however, it may be appropriate to specify more outcomes of interest beyond improved competencies, engagement and literacy to possibly include addressing actual health behaviors or behavioral intentions.

A potential scenario for ME:MIM is that this approach to teaching and learning may be adapted and integrated as an independent or integral component of existing health education curricula in schools, or as a stand-alone elective course, perhaps in high school. Should this scenario be pursued, it would heighten the need for process and outcome evaluation studies to assess intervention integrity. Low implementation integrity is a potential problem with new interventions and suggests at least one of the following considerations: a) the intervention needs to be modified; b) those providing the intervention need additional training; or c) additional implementation support and provider accountability related to quality implementation of the intervention is needed. One clear benefit of implementation integrity is that it provides an additional measure of feasibility. Identifying sources of fidelity lapses will enable the refinement of intervention components. Developing explicit training procedures is very important to any future ME:MIM research-based scenarios.

\section{Future research}

As noted in the literature reviewed, Weissberg and Greenberg [38] urged that competence be viewed and measured in research studies as a stand-alone developmental outcome. Importantly, research that 
suggests the enhancement of competence can help prevent other negative outcomes in youth [39]. Perhaps of even greater importance is the fact that competence can be specified and measured independently serving as its own outcome indicator of positive youth development.

A next step in this program of research is to modify ME:MIM based on findings from this study and prepare to launch a follow-up study that will likely focus on staff training based on the creation of a manual that identifies outcome measures. In the interim, this will require developing instructional support resources such as a song database tagged with themes in popular youth music media. This technologyenabled resource will facilitate and standardize instructional materials that assist students and teachers in making the link between music, perceptions, behavior, cognition, values, choices and outcomes [40-44].

Another goal in a future research scenario is to determine the effectiveness of the intervention via a randomized controlled trial. An intervention's effectiveness should be assessed by how well it addresses its goals. The goals of ME:MIM are: a) to engage students in social learning; b) to conduct instruction and enrichment activities that address national and state-adopted standards aligned with health education and media literacy; and c) to reinforce competencies linked to positive youth development and school success. While the specific skills and abilities addressed in this intervention are education-related, they are also required skills all adolescents must master to sustain their health and wellbeing. Evidence of these skills at the individual or group level of analysis would go a long way toward informing policy makers and practioners of the utility of such an approach. Determining the effectiveness of smaller components of the intervention at the modular level could also yield important insight that may strengthen the case for using popular youth culture and popular youth music media to clarify values linked to education, health and behavior $[45,46]$.

\section{References}

1. Fischer P, Greitemeyer T, Kastenmüller A, Vogrincic C, Sauer A (2011) The effects of risk-glorifying media exposure on risk-positive cognitions, emotions, and behaviors: a meta-analytic review. Psychol Bull 137: 367-390.

2. Anderson CA, Douglas AG (2014) Violent Video Game effects on Aggressive thoughts, feelings, physiology, and Behavior. Media Violence and Children: A Complete Guide for Parents and Professionals, p: 229.

3. Austin EW, Pinkleton BE, Hust SJ, Cohen M (2005) Evaluation of an American Legacy Foundation/Washington State Department Of Health Media Literacy Pilot Study. Health Commun 18: 75-95.

4. Bergsma LJ, Carney ME (2008) Effectiveness of health-promoting media literacy education: a systematic review. Health Educ Res 23: 522-542.

5. IML (2010) Bergsma Media Literacy into your Curriculum.

6. David T (1984) The rap attack: African jive to New York hip hop. Boston, South End Press.

7. Khalil SP, Woods TP (2014) Hip Hop Studies in Black. Journal of Popular Music Studies 26: 268-294.

8. Harper H, Thandi P (1993) Black educators, black elementary school students, and black rap music artists on educational entertainment rap music video for pedagogy: a cultural and critical analysis.

9. Victoria RJ, Foehr UG, Roberts DF (2010) Generation M: Media in the Lives of 8- to 18- Year-Olds. Henry J. Kaiser Family Foundation.

10. Eaton DK, Kann L, Kinchen S, Shanklin S, Flint KH, et al. (2012) Youth risk behavior surveillance - United States, 2011. MMWR Surveill Summ 61: 1-162.

11. Molina BS, Smith BH, Pelham WE Jr (2005) Development of a schoolwide behavior program in a public middle school: an illustration of deployment-focused intervention development, stage. J Atten Disord 9: 333-342.
12. John RW (2004) Psychotherapy for children and adolescents: Evidencebased treatments and case examples. Cambridge University Press.

13. Kelly SN (2015) Teaching music in American society: A social and cultural understanding of music education. Routledge.

14. Crossan C, Mary M (1995) Organizational learning: Dimensions for a theory. The International Journal of Organizational Analysis 3: 337-360.

15. Bandura A (1989) Human agency in social cognitive theory. Am Psychol 44: 1175-1184.

16. Scott SM, Wallander JL, Cameron L (2015) Protective Mechanisms for Depression among Racial/Ethnic Minority Youth: Empirical Findings, Issues, and Recommendations. Clin Child Fam Psychol Rev 18: 346-369.

17. Frieden P, Thomas R (2005) BRFSS is an annual state-based randomdigit-dialed telephone (landline and cell phone) survey of the US non institutionalized civilian population.

18. Posner JK, Vandell DL (1994) Low-income children's after-school care: are there beneficial effects of after-school programs. Child Dev 65: 440-456.

19. Harper H, Hicks PT (2000) Understanding youth popular culture and the hip-hop influence. Siecus Report 28: 19.

20. Media Literacy Now (2015) Media Literacy.

21. Balfanz R, Joanna F (1977) Sent home and put off-track: The antecedents, disproportionalities, and consequences of being suspended in the ninth grade. Journal of Applied Research on Children: Informing Policy for Children at Risk 5: 13.

22. Cantor J (2003) Media violence effects and interventions: The roles of communication and emotion. Communication and emotion: Essays in honor of Dolf Zillmann, pp: 197-220.

23. Paul C, Coghlan D (2002) Action research for operations management. International journal of operations \& production management 22: 220-240.

24. Zais M (2011) South Carolina Grade Level Health and Safety Education Curriculum Guidelines.

25. Patton P, Quinn M (2002) Two decades of developments in qualitative inquiry a personal, experiential perspective. Qualitative social work 1: 261-283.

26. Norman DK, Yvonna S, Lincoln K (2008) Strategies of qualitative inquiry. Sage.

27. Miles M, Matthew B, Michael HA (1994) Qualitative data analysis: An expanded sourcebook. Sage.

28. Anselm S (1998) Strategies of qualitative inquiry. pp: 158-183.

29. Dey I (1999) Grounding grounded theory: Guidelines for qualitative inquiry. Academic Press.

30. Andrew SC (2005) Narrative analysis: exploring the whats and hows of personal stories. Qualitative research in health care 1: 191-209.

31. Pitman MA, Joseph A (1992) Qualitative approaches to evaluation: Models and methods. The handbook of qualitative research in education. Maxwell, pp: 729-770.

32. Marshall C, Gretchen BR (2014) Designing qualitative research. Sage publications.

33. Gardner H, Thomas H (1989) Educational implications of the theory of multiple intelligences. Educational researcher 18: 4-10.

34. James JA (1996) Vygotsky's sociocultural theory and contributions to the development of constructivist curricula. Education 117: 133.

35. Bandura A (2011) Social learning theory.

36. Piaget J (1971) The theory of stages in cognitive development.

37. Keiichi T (2008) Jerome Bruner's theory of education: From early Bruner to later Bruner. Interchange 39: 1-19.

38. Roger PW, Greenberg MT (1998) School and community competenceenhancement and prevention programs.

39. Botvin GJ (1995) Effectiveness of culturally focused and generic skills training approaches to alcohol and drug abuse prevention among minority adolescents: two-year follow-up results. Psychology of Addictive Behaviors 9: 183. 
Citation: Owens JD, Smith BH (2016) Health Education and Media Literacy: A Culturally-Responsive Approach to Positive Youth Development. J Health Edu Res Dev 4: 169. doi:10.4172/2380-5439.1000169

Page 12 of 12

40. Adams CD, Nancy H, Gerard GR (1994) Behavioral difficulties in toddlers: Impact of socio-cultural and biological risk factors. Journal of Clinical Child Psychology 23: 373-381.

41. Erik H (1994) Identity: Youth and crisis. Psychology, p: 336.

42. Forehand M (2010) Bloom's taxonomy. Emerging perspectives on learning, teaching, and technology, pp: 41-47.

43. Helding L (2009) Howard Gardner's theory of multiple intelligences. Journal of Singing 66: 193.
44. http://education.jhu.edu/PD/newhorizons/lifelonglearning/adolescence

45. Peter R, Bradbury H (2001) Handbook of action research: Participative inquiry and practice. Sage.

46. Barbara JW (2002) Violence in children's television programming: Assessing the risks. Journal of Communication 52: 5-35. 